\title{
Spectral and Osmotic Fragility Analysis of Erythrocytes for Neonatal Jaundice
}

\author{
Azza Nady M. H. Department of Basic Sciences, Higher \\ Technological Institute, $10^{\text {th }}$ of Ramadan City, Al-Sharqiyah, \\ Egypt.
}

\begin{abstract}
T EONATAL jaundice( physiological jaundice), an extremely common occurrence among neonates in the first week of life, can represent a benign physiological process or be the harbinger of serious illness with associated severe neurotoxicity. In the present work, a group of sixty five infants (GpI: control group, GpII: jaundiced neonates, GpIII: Glucose 6 phosphate dehydrogenase (G-6PD) deficient neonates and GpIV: is the Crigler -Najjar I (CNI) and Crigler -Najjar II (CNII) neonates), were included.The rheological and durability properties of the erythrocytes of jaundiced neonates were investigated using spectral analysis and osmotic fragility. The results revealed that, there was a significant decrease in the mean cell fragility (MCF) from 0.41 for control cases to 0.298 for CNI case also the hemolytic ratio shows a sharp decay at G-6-PD conc. $<4 \mathrm{U} / \mathrm{gHb}^{*}$. Absorption spectra of erythrocytes shows a significant decrease of splitting factor (SF) as bilirubin (Bb) conc. increases, indicating that the cells tend to convert from oxygenated to deoxygenated state with a peak at $560 \mathrm{~nm}$ which is confirmed by SF values. It may be concluded that a severe hyperbilirubinemia may leads to irreversible damage of spheroechinocyte cells, causing cell lysis .
\end{abstract}

Keywords: Neonatal jaundice, G-6-PD, Bilirubin, Osmotic fragility, Spectral analysis.

Bilirubin is the principal degradation and product of heme moiety from hemoglobin. Features of pathologic jaundice include a total serum bilirubin level $>17 \mathrm{mg} / \mathrm{dl}$ in a full-term newborn ${ }^{(1)}$. The increased bilirubin production depends on produced erythrocytes life span in the neonate and the defective bilirubin uptake by immature liver.

Like other cell types, erythrocytes are capable of binding unconjugated $\mathrm{Bb}$ to their membrane as demonstrated by several studies conducted in vivo and in vitro.The toxic effect of $\mathrm{Bb}$ was demonstrated to result from its association with membrane lipids. Brito et al. ${ }^{(2)}$ showed that the cholesterol / phospholipid $(\mathrm{ch} / \mathrm{pl})$ ratio is higher and $\mathrm{pl}$ content was lower in jaundiced neonates compared with healthy babies. G-6-PD deficiency has been associated with severe neonatal hyperbilirubinemia and kernicterus ${ }^{(3)}$. The predominant factor in pathogenesis of neonatal hyperbilirubinemia (NHB) associated with G-6-PD deficiency has been shown to be a decreased bilirubin conjugation.

*One international unit (U) is that amount of G-6-PDH activity that will convert 1 micromole of substance per minute. 
$\mathrm{CNI}$ is recessively inherited, potentially lethal disorder characterized by severe unconjugated hyperbilirubinemia ${ }^{(4)}$. CNI and CNII were diagnosed, using polymerase chain reaction (PCR) technique to identify the sequence determination of exons encoding bilirubin-uridinediphosphoglucronosyltransferase (B-UGT1 and B-UGT2).The red blood cells (RBCs) are more common than other blood cells and carry information about donor health conditions ${ }^{(1)}$. The human RBCs can be used as a model to study unconjugated bilirubin cellular binding and toxicity due to its accessibility.The ability of normal red cell to withstand hypotonicity results from its biconcave shape which allows the cell to increase its volume by about $70 \%$ before the surface membrane is stretched, this is occurred in normal RBCs, while in case of hyperbilirubinemiadue to jaundiced neonates or infant with G-6-PD deficiency, this property is not present. UV-vis absorption spectroscopy is one of the simplest, label techniques yielding direct insight into electronic transitions. It's particularly sensitive to the binding of oxygen by hemoglobin and, therefore, has been used to monitor oxygenation state transitions in both bulk hemoglobin solutions and more recently, in single RBCs ${ }^{(5)}$. Studying RBCs deformations for Jaundiced neonates and effect of G-6-PD deficiency are necessary in understanding the fragility of RBCs by using spectral and osmotic fragility analysis.

\section{Materials and Methods}

\section{Materials}

Sample preparation

This study was conducted at Kasr Al-Aini Hospital after the approval of Pediatric Department (Faculty of Medicine, Cairo University) and the local Ethics Research Committee. Infants were consecutively enrolled if informed consent had been given by their parents.

A group of 65 infants (20 controls +45 patients) were included in present study.

They were divided into 4 groups:

- Group I (Gp I) is the control group with an average value of Bb concentration equal to $1.5 \pm 0.4 \mathrm{mg} / \mathrm{dl}$.

-Group II (Gp II) is the jaundiced neonates with an average value of $\mathrm{Bb}$ concentration equal to $20.6 \pm 8.7 \mathrm{mg} / \mathrm{dl}$. Vinous blood samples from 24 (15 male and 9 female) jaundiced neonates was collected between $6^{\text {th }}$ and $12^{\text {th }}$ days of life.

-Group III (Gp III) is the G-6-PD deficient neonates with an average value of G6-PD concentration equal to $2.85 \pm 0.7 \mathrm{U} / \mathrm{gHb}$. This group include 19 (11 male and 8 female) infants.

-Group IV (Gp IV) is the CNI and CNII neonates. This group includes two female infants, one has CNI and the other infant has CNII. They were diagnosed as CNI and CNII using PCR technique ${ }^{(6)}$. 
TABLE 1. Demographic characteristic of 63 neonates enrolled in this study.

\begin{tabular}{|l|c|c|c|}
\hline Category & GP I $(\mathbf{n = 2 0})$ & GP II $(\mathbf{n = 2 4})$ & GP III (n=19) \\
\hline Female/Male & $13 / 7$ & $15 / 9$ & $11 / 8$ \\
\hline Gestational age (week) & $39.7 \pm 1$ & $39.1 \pm 1.8$ & $38.6 \pm 1.2$ \\
\hline Weight & $3750 \pm 220$ & $3310 \pm 72$ & $3100 \pm 560$ \\
\hline Jaundice appearance (days) & control & $2.4 \pm 0.5$ & $5.2 \pm 2.2$ \\
\hline
\end{tabular}

The exclusion criteria were pathological hyperbilirubinamia such as $\mathrm{Rh}$ or $\mathrm{ABO}$ is immune hemolytic anemia, cephalohematoma or sepsis.

\section{Methods}

$U V$ - vis. Spectral analysis

Blood samples were obtained from healthy and jaundiced neonates with strict adherence to the ethical guidelines. Blood was collected in heparinized tubes and centrifuged for $10 \mathrm{~min}$ at $6000 \mathrm{rpm}$ at $4^{\circ} \mathrm{C}$. Samples were washed three times with a phosphate buffered saline (PBS: Nacl $150 \mathrm{~m} \mu, \mathrm{KH}_{2} \mathrm{Po}_{4} 0.58 \mathrm{~m} \mu$ and $\mathrm{Na}_{2} \mathrm{HPo}_{4}$ $3.4 \mathrm{m \mu}, \mathrm{pH}$ 7.4). Plasma and buffy coat were carefully removed by aspiration after each washing. The settled precipitate was diluted with buffered solution and stored at $4^{\circ} \mathrm{C}$. Hemolysis of RBCs was carried out by mixing $5 \%$ of RBCs in PBS then incubate for $3 \mathrm{~h}$ at $37^{\circ} \mathrm{C}$ with gentle shaking. The extent of hemolysis was determined spectrophotometrically by measuring the absorbance of hemolysate at $540 \mathrm{~nm}$.

\section{Measurement of cellular osmotic fragility}

RBCs were incubated in solutions with various concentrations of $\mathrm{Nacl}$ (range from $10 \%$ to $85 \%$ ) for $30 \mathrm{~min}$. After centrifugation, the supernatants were analyzed at $540 \mathrm{~nm}$ in a spectrophotometer. The hemolytic ratio $\left(\mathrm{H}_{\mathrm{r}}\right)$ was calculated by the following formula:

$$
H_{r}=\frac{T_{r 300}-T_{r}}{T_{r 300}-T_{r 40}} \times 100 \%
$$

where:

$T_{r 40}$ is the transmittance at concentration of $(0.1 \% \mathrm{Nacl})$.

$T_{r 300}$ is the transmittance at concentration of $(0.85 \% \mathrm{Nacl})$.

\section{Results}

Osmotic fragility

The results of RBCs osmotic fragility for control, G-6-PD deficiency, an ordinary jaundiced neonates and severe hyperbilirubinemia (CNI case), show that the hemolysis curve of CNI shifts to the left more than G-6-PD deficiency and Neonatal jaundice (NJ) cases (Fig. 1). Also, the same result was fitted as shown in Fig. 2. There is a significant decrease in the mean corpuscular fragility (MCF) from 0.41 for control case to 0.298 for CNI case, also, there is a shift to 
lower MCF for G-6-PD def. and NJ cases to be 0.388 and 0.364 , respectively. The results show also a decrease in the hemolytic ratio as a function of $\mathrm{Bb}$ concentration till certain concentration of $\mathrm{Bb}$ (around $15 \mathrm{mg} / \mathrm{dl}$ region) followed by some increase in hemolytic ratio after this range as shown in Fig. 3.

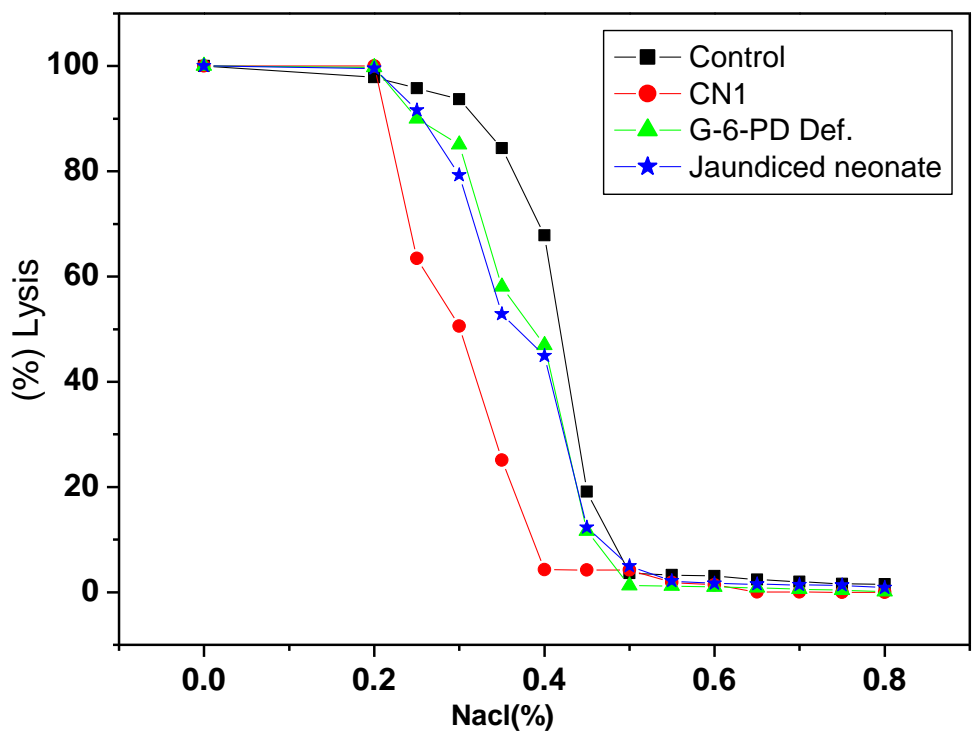

Fig. 1. Osmotic fragility curves for control, CNI case, G-6-PD deficiency and jaundiced neonates.

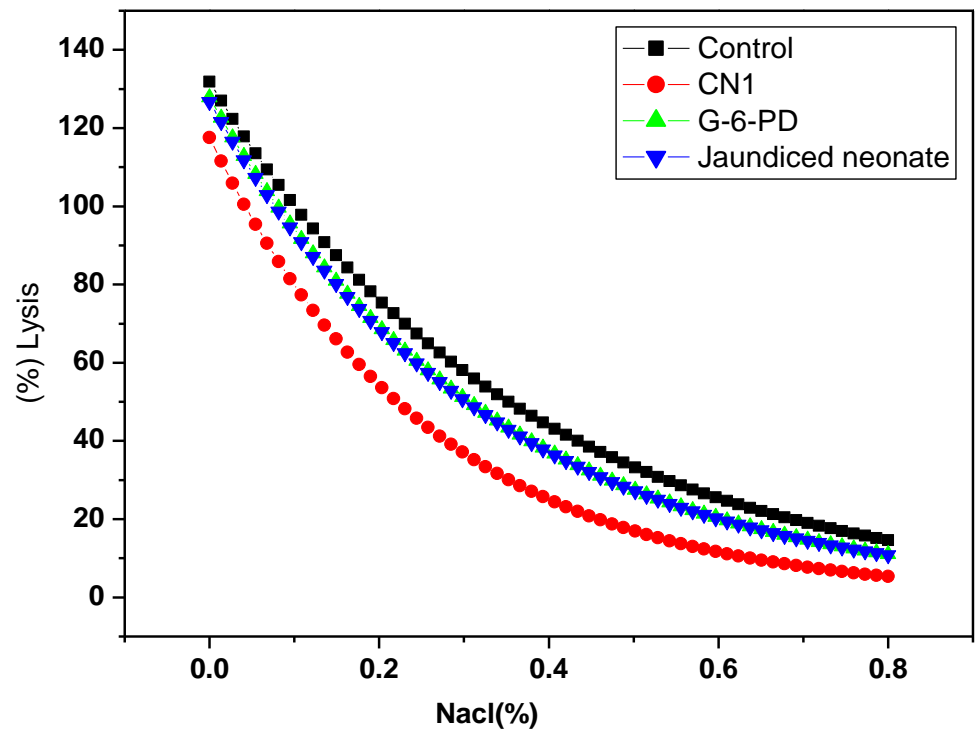

Fig. 2. Exponential decay fitting for the data of Fig. 1 indicate the degree of hemolysis for control and different Jaundiced neonates.

Egypt. J. Biophys. Biomed. Engng. Vol. 13 (2012) 


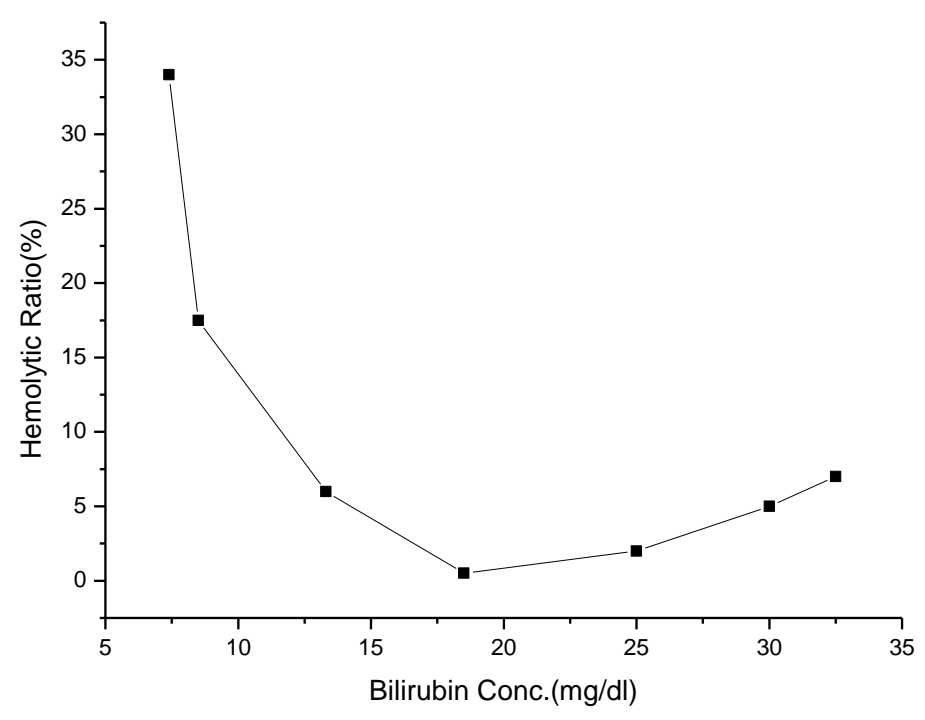

Fig. 3. Hemolytic ratio of RBCs curve at different $\mathrm{Bb}$ concentration.

In the G-6-PD deficiency group as shown in Fig. 4 the hemolytic ratio shows a sharp decay at lower value of G-6-PD concentration $(<4 \mathrm{U} / \mathrm{g} \mathrm{Hb})$.

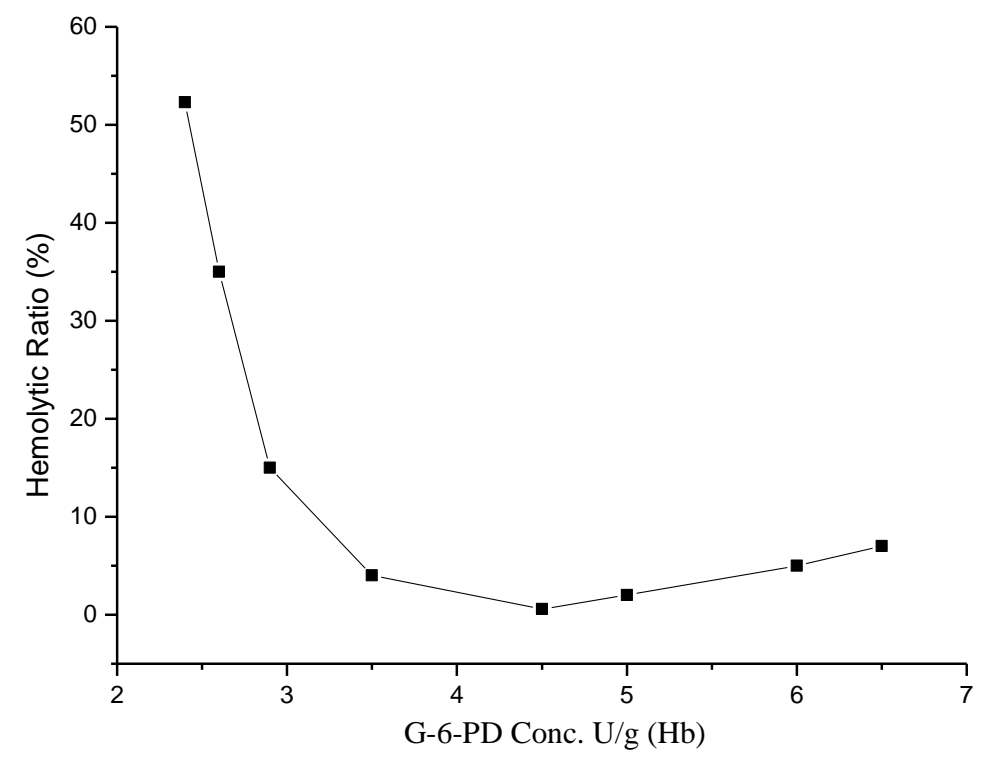

Fig. 4. Hemolytic ratio of RBCs curve at different G-6-PD deficiency concentration. 


\section{Absorption spectra}

Figure 5 shows the results of UV-vis. absorption spectra of erythrocytes for control, G-6-PD deficiency, an ordinary jaundiced neonate, and CNI case. The spectra show the soret band located at $410 \mathrm{~nm}$ followed by spectra of oxy and de-oxy forms of $\mathrm{Hb}$ which are dominated at $575 \mathrm{~nm}$ and its repetition at about wavelength of $540 \mathrm{~nm}$.

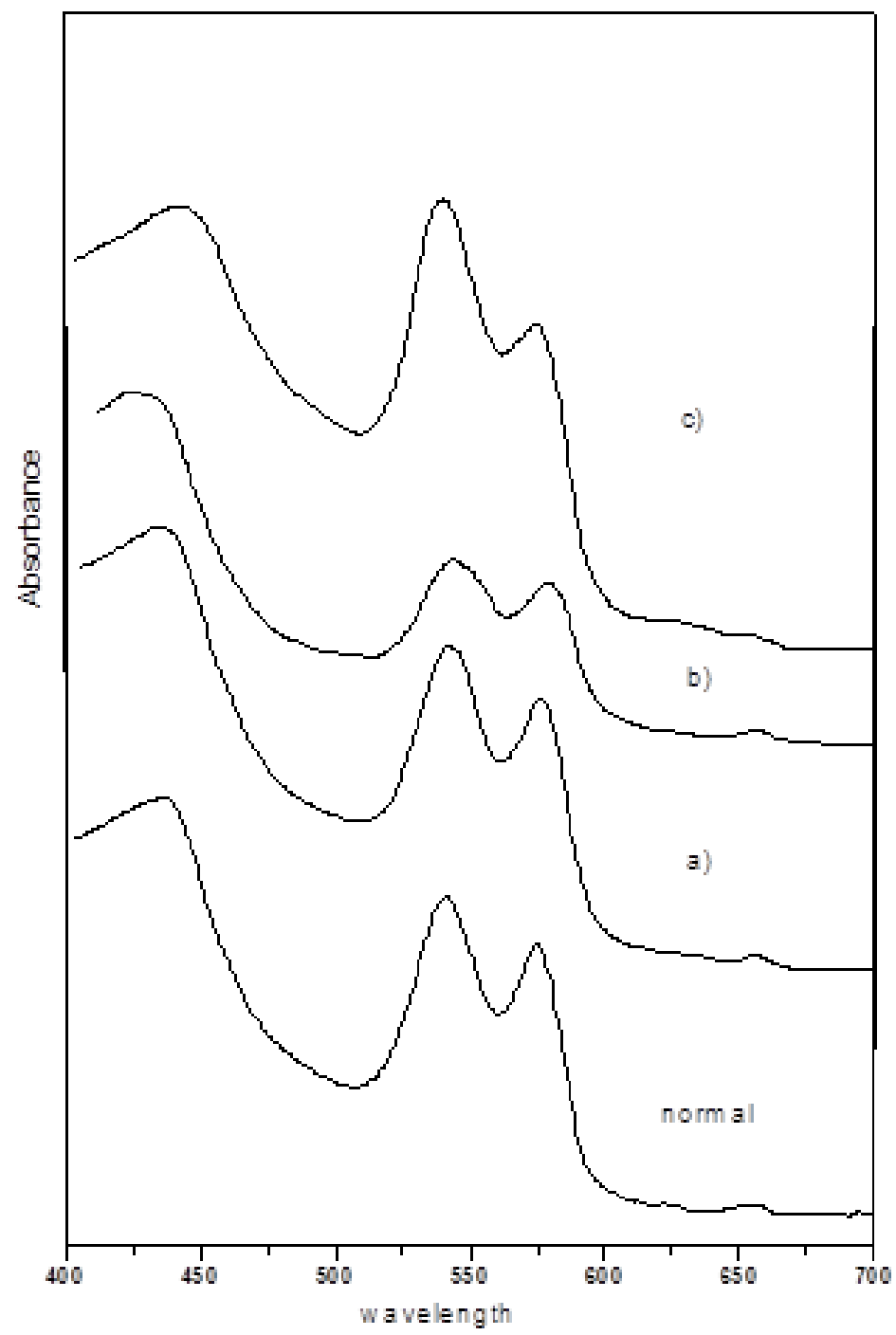

Fig. 5. Absorption spectra of RBCs in soret and heme-heme interaction for control, (a) G-6-PD deficiency, (b) NJ and (c) CNI cases. 
Figure 5 shows that a significant decrease of splitting factor $\left(\frac{A_{540}+A_{575}}{A_{560}}\right)$ that is induced due to an increase in bilirubin concentration, and there is a higher ratio $\frac{A_{560}}{A_{575}}$ at this splitting factor. This indicates that the cells tend to convert from oxygenated to deoxygenated state.

\section{Discussion}

The effect of bilirubin on RBC's morphology has been studied and quantified by Anwar et $a l .{ }^{(6)}$.In the present study an enhancement in osmotic fragility of erythrocytes was observed for G-6-PD deficiency neonates and CNI patient. Both cases emphasized heterogenity of the cells with respect to osmotic fragility. The higher free bilirubin concentration tends to approach the irreversible hyperbilirubinemia which lead to pathological condition of kernicterus $(\mathrm{CNI})^{(2)}$. Among preterm infants there is a relationship between water loss and exposure to the light during phototherapy ${ }^{(7)}$ which leads to an increase of hemoglobin concentration, i.e. for Crigler -Najjar patient, exposure for a long time to the light causes dehydration and severe echinocytosis.This explains the relation between the cell lysis and $\mathrm{NaCl}$ concentration as shown in Fig.1. Erythrocyte membrane oxidation causes membrane rigidification and increased resistance to shape change, so the RBC's will have short life span ${ }^{(8)}$.

The blood as well as the biological tissues represents a special class of heterogeneous systems. The change in the hemolytic ratio $(\mathrm{Hr})$ of $\mathrm{RBC}$ 's reflects changes occurring in erythrocytes due to change in bilirubin and G-6-PD difficiency. At bilirubin concentration $>15 \mathrm{mg} / \mathrm{dl}$ unbound unconjugated bilirubin (free bilirubin) cause sufficient stress on RBC's membrane, which transform the erythrocyte into smooth sphere which rapidly hemolysis ${ }^{(9)}$ as shown in Fig. 3. This figure shows some increase in hemolytic ratio at bilirubin concentration > $15 \mathrm{mg} / \mathrm{dl}$, where a very fragile cells are present at this concentration, with small cell surface area to volume ratio, forming a spheroechinocytic cells at this region and this result in agreement with that obtained by $\mathrm{Nady}^{(10)}$. The alteration in the red cell permeability barrier causes water entrance to the cell followed by cell swelling and ultimately colloid osmotic hemolysis ${ }^{(11)}$. In G-6-PD deficient erythrocytes, the shunt activity is significantly decreased. The oxidation effects of the hexose monophosphate (HMP) shunt activators couldn't increase shape recovery rates, so that at this lower concentration of G-6$\mathrm{PD}$, there is a sharp drop of hemolytic ratio due to dramatic effect (cell lysis) on erythrocytes as shown in Fig. 4. Erythrocyte deformability is an important index of blood flow and oxygen transport in microcirculations ${ }^{(12)}$.

Therefore, it is thought that the erythrocyte membrane has a change in $\mathrm{ch} / \mathrm{pl}$ ratio that cause alterations in membrane lipid-protein interactions, together with 
increased abnormal hemoglobin concentration (Heinz bodies due to presence of hemoglobin under oxidative stress) ${ }^{(2)}$, and how the RBC's deformations induced by different concentrations of bilirubin and G-6-PD may change the RBC's absorption spectra.

The heme groups embedded in a hydrophobic protein backbone with characteristic conformation are the main reason for the measured absorption bands. The difference in the absorption among these cells can be explained by the variation of cell morphology and $\mathrm{Hb}$ concentration. Fore example, the high absorption at $\lambda_{560}$ for jaundiced neonate erythrocytes indicates that the cells have reversible morphological changes, and these cells tends to be in deoxygenated form for CNI, the cells break down (sphero-echinocyte cells) and the rest of cells are in deoxygenated form with lower absorbance at the same band.

The present study shows that the intensity ratio of deoxy peaks to that of oxy peaks $\left(A_{560} / A_{575}\right)$ increases in erythrocytes of CNI. Figure 5 indicates a gradual decrease in splitting factor $\left(\frac{A_{540}+A_{575}}{A_{560}}\right)$ for control, G-6-PD deficiency, $\mathrm{NJ}$ and CNI patients.

At CNI case there is a significant increase in the absorbance at $540 \mathrm{~nm}$ and lower intensity at wavelength $\lambda_{575}$ (it looks like a shoulder), which comes in agreement with morphological study performed by Anwar et al. ${ }^{(6)}$.The changes in the shape and position of absorption bands could therefore indicate possible conformational changes of the surrounding proteins ${ }^{(13)}$. Also, in the soret region there is a significant shift of the absorption band towards higher wavelength, this shift appears for jaundiced neonate ( at $421 \mathrm{~nm}$ ), then reaches maximum for CNI case ( at 444nm). This shift leads to strong inhomogeneity. The soret band is due to the presence of $\mathrm{Hb}$ in its native form and it is very sensitive to alterations in the surrounding environment ${ }^{(13)}$.

\section{Conclusion}

In the present work, it may be suggested that :

-Morphological alterations of erythrocytes for jaundiced neonates are strongly dependent on Bb and G-6-PD deficiency (causing hyperbilirubinemia).

-The degree of oxy $\rightarrow$ deoxy cell conversion with a peak at $560 \mathrm{~nm}$ which confirmed by degree of change in splitting factor (SF) may leads to perturbations of membrane skeletal protein network.

- Hyperbilirubinemia gives rise to membrane instability and leads to reduction of cell surface area, then the cell deformability and cell lysis. This means that severe hyperbilirubinemia due to G-6-PD defficiency or neonatal jaundice should be responsible for the irreversible damage that leads to cell lysis.

Egypt. J. Biophys. Biomed. Engng. Vol. 13 (2012) 
- Bilirubin conc. at $15 \mathrm{mg} / \mathrm{dl}$ is a critical conc. where at Bb conc.> $15 \mathrm{mg} / \mathrm{dl} \mathrm{a}$ very fragile cells are present.

- G-6-PD conc. at $4 \mathrm{U} / \mathrm{gHb}$ shows a dramatic change of hemolytic ratio due to cell lysis on erythrocytes.

\section{References}

1. Brito, M., Silva, R., Matos, D. and Brites, D., Bilirubin toxicity to human erythrocyte: A review. Clinica Chimica Acta, 374, 46-56 (2006).

2. Brito, M., Silva, R., Matos, D., da Silva, A. and Brites, D., Alterations of erythrocyte morphology and lipid composition by hyperbilirubinemia. Clinica Chimica Acta, 294, 149-165 (1996) .

3. Mac Doland, M.G., Hidden risks: Early discharge and bilirubin toxicity due to glucose 6-phosphate dehydrogenase deficiency. Pediatrics, 96, 734 - 738 (1995).

4. Behrman, R., Kliegman, R. and Jenson, H. "Text book of Pediatrics" Nelson, (Ed). $16^{\text {th }}$ ed. Saunders, ch. 357 (2000).

5. Michal, W., Sauabh Raj and Dmitri, P., Absorption spectroscopy of single red blood cells in the presence of mechanical deformations induced by optical traps, Journal of Biomedical Optics, 17(9), 1-8 (2012).

6. Anwar, M. Ali., Kholo Naglaa, M., Ezz El- Din, Zahraa M. Nadi, Azza and Abdel Aleem, Alice K., Neonatal jaundice and its association with erythrocytes deformation and molecular changes. The Egyptian Journal of Laboratory Medicine, 431- 444 (2004).

7. Grunhagen, D., de Boer, M., de Beaufort, A. and Walther, F., Transepidermal water loss during halogen spotlight phototherapy in preterm infamts. Pediatr. Res. 51 (3), 402-5 (2002).

8. Fanaroff, A. and Martin, R., "Neonatal Perintal Medicine, Diseases of The Fetus and Infant", Vol. 2, $6^{\text {th }}$ ed., Ch.45 in : Neonatal jaundice and liver disease by Halam, L. and Steveson, D. (1996).

9. Wong, P., A basis of echinocytosis and stomatocytosis in the discspheretransformations of the erythrocyte. J. Theor. Biol. 196, 343-361 (1999).

10. Nady, Azza M., Electrical and morphological changes of erythrocytes of jaundiced neonates followed by dielectric spectroscopy, Al-Azhar Bull. Sci. (ISCAZ,) 123-142 (2010).

11. Beutler, E., Marshalla, M., Lichtman, M., et al., "Hematology", $6^{\text {th }}$ ed. (2001).

12. Tsukada, K., Sekizuka, E., Oshio, C. and Minamitani, H., Direct measurement of erythrocyte deformability in diabetes mellitus with transparent microchannelcapillary model and high - speed video camera system. Microvascular Res. 61, 231-239. (2001). 
13. Wojdyla, M., Raj, S. and Petrov, D., Absorption spectroscopy of single red blood cells in the presence of mechanical deformations induced by optical traps. $J$. Biomedical Optics, 17(9) 1-8 (2012).

\title{
التحليل الطيفي والوهن الاسموزي لكرات الام الحمراء عند الاطفـال حايثي الولاده المصابيين بالصفراء الأوني
}

\author{
عزه نادى محمد حمزه \\ معهد التكنولوجيا العالي بالعاشر من رمضدانـ محافظة الثرقيهـ مصر.
}

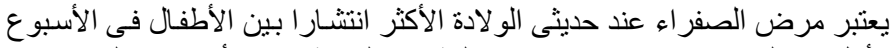

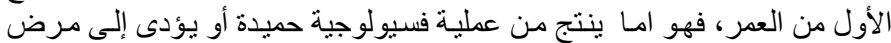

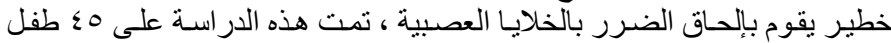

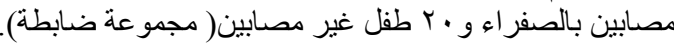

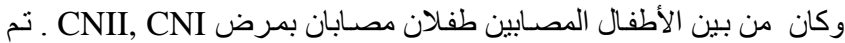

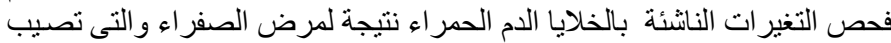

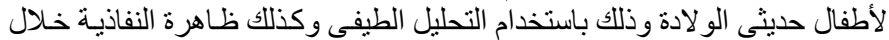

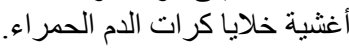

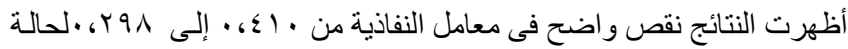

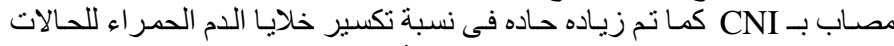

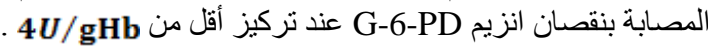

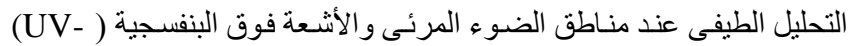

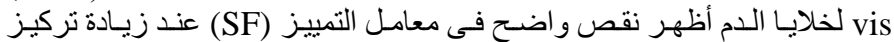

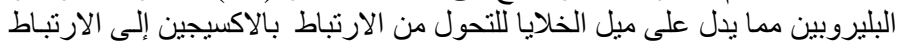

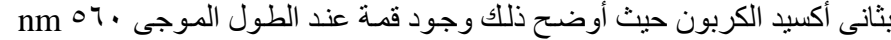

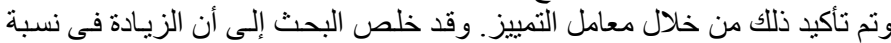

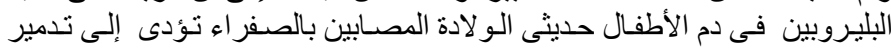

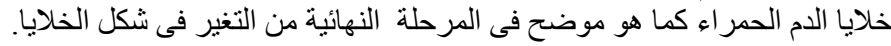

